\section{Synthesis and Characterization of Carrageenan Coated Magnetic Nanoparticles for Drug Delivery Applications}

\author{
Shanmuga SI, Singhal M and \\ Sen S \\ School of Bioscience and Technology, VIT \\ University, Vellore, Tamil Nadu, India
}

Corresponding author: Prof. Shampa Sen

\section{Abstract}

Background: Nanomaterials are extensively used from household applications to industrial applications. Nanomaterials are the substances that are synthesized in the size ranging from 1-100 $\mathrm{nm}$ which exhibits excellent physical and chemical properties compared to their bulk forms. Iron oxide nanoparticles (magnetic) have unique properties that make them applicable in drug delivery applications. However due to the higher oxidation state of iron, those nanoparticles possess less stability and tends to agglomerate in the body when used for such applications. This paper reports on the synthesis of magnetic nanoparticles and coating them with natural polymer, carrageenan to avoid agglomeration.

Methods and findings: Carrageenan was extracted from red algae obtained from Rameswaram. Pure carrageenan was obtained by ethanol precipitation method. The obtained polymer was used for synthesis of magnetic nanoparticles by coprecipitation technique. The synthesized polymer coated magnetic nanoparticles were characterized by various characterization techniques and was found to possess the characteristics of being nanoparticle. The obtained nanoparticle was checked for its anti-haemolytic activity as an initial screening for drug delivery applications

Conclusion: The synthesized nanoparticle can be widely used for drug delivery applications as carrageenan, natural polymer has advantages compared to synthetic polymers.

Keywords: Magnetic nanoparticles; Drug delivery; Nanomaterials; Carrageenan; Nanotechnology

\section{” shampa.iitg@gmail.com}

Associate Professor, School of Bioscience and Technology, VIT University, Vellore - 632 014, India.

Tel: +91 9790493264

Fax: 91-416-2243092

Citation: Shanmuga SI, Singhal M, Sen S. Synthesis and Characterization of Carrageenan Coated Magnetic Nanoparticles for Drug Delivery Applications. Transl Biomed. 2015, 6:3.

Received: September 01, 2015; Accepted: November 10, 2015; Published: November 13, 2015

\section{Introduction}

One of the emerging technologies in today's world is Nanotechnology. The concept of engineering nanomaterials which are in the scale of 1-100 $\mathrm{nm}$ were applied in almost all the fields; from food to biomedical applications. Materials engineered at nano size possess unique properties compared to their bulk forms. Nano sized particles holds different properties such as magnetism, fluorescence, photo degradation, high surface to charge ratio etc. which made the human society to apply nanomaterials for even basic applications like floor cleaner and nano-socks. Tremendous applications can be found in biomedical applications. The exceptional properties of nanomaterials are greatly applied in drug delivery applications. Such application focus mainly to overcome the problems existing in drug delivery approaches. The major glitches observed in drug delivery applications are lower bioavailability, biocompatibility, adverse side effects, toxicity and failure to reach the target site. All these problems can be overwhelmed by using nanoparticles.

Iron oxide nanoparticles are non-toxic particles which can be utilized by the body during hemoglobin metabolism; hence they are reusable and non-toxic. Iron oxide nanoparticles exist in three different forms; magnetite $\left(\mathrm{Fe}_{3} \mathrm{O}_{4}\right)$, maghemite $\left(y\right.$ - $\left.\mathrm{Fe}_{2} \mathrm{O}_{3}\right)$ and hematite $\left(\alpha-\mathrm{Fe}_{2} \mathrm{O}_{3}\right)$ [1]. Of the three, magnetic nanoparticles are often used for drug delivery applications due to their unique magnetic properties. However, due to the higher oxidation of iron, magnetic nanoparticles possess less stability. Hence to get 
magnetic nanoparticles of good stability with desired size and shape, MNPs are coated/conjugates with polymers.

Polymers are synthetic or naturally derived materials used as stabilizing agents or surfactants like polyethylene glycol, tween 80 respectively. MNPs are functionalized with various polymers as polymer renders higher bioavailability and non-toxicity. There are several reports on coating of nanoparticles with natural polymer dextran, alginate, chitosan, starch based coatings and K-carrageenan [2]. This paper reports on carrageenan, a natural polymer extracted from sea weed. Carrageenan are sulphated linear polysaccharides exists in different forms prefixed with lota (i-), Kappa (j-) and Lambda (k)-carrageenan [3]. K-carrageenan are the predominant polymer of red algae kappaphycus alvarezzi; tough and fleshy red algae. Kappaphycus spp. is widely found on any reef flats and reef edges that occur from almost 1 to $17 \mathrm{~m}$ deep.

\section{Methods}

\section{Carrageenan isolation and extraction}

The red algae Kappaphycus alvarezzi was shade dried for 15 days and grinded using regular mixture grinder to get a fine powder. About 5 gms of the powdered sample was added to $500 \mathrm{ml}$ of $0.05 \mathrm{M} \mathrm{KOH}$ alkali solution, kept in water bath for 3 hours at $80^{\circ} \mathrm{C}$ for boiling to extract carrageenan. The solution was filtered, centrifuged at $10,000 \mathrm{rpm}$ for $10 \mathrm{~min}$ at $30^{\circ} \mathrm{C}$ to remove the minute cell debris. The obtained hot crude carrageenan solution was precipitated with ice cold $100 \%$ ethanol at $4-6^{\circ} \mathrm{C}$ to get pure carrageenan gel. The carrageenan gel was presses in a sieve to remove excess ethanol and the gel was dried at 60 o $\mathrm{C}$ in hot air oven for 12 hours to get dried carrageenan powder. The powder was dissolved in deionized water and used for experimental purposes.

\section{Synthesis of magnetic nanoparticles}

Magnetic nanoparticles are synthesized by Co-precipitation technique [4]. Salts of $\mathrm{Fe}^{2+}$ and $\mathrm{Fe}^{3+}$ are added in a desired stoichiometric ratio and are precipitated at alkaline $\mathrm{pH}$ to get desired $\mathrm{Fe}_{3} \mathrm{O}_{4}$ MNPs as shown below. Thermodynamically, precipitation of MNP occurs in $\mathrm{pH}$ ranging from 8 to 14 with stoichiometric ratio of $2: 1\left(\mathrm{Fe}^{3+} / \mathrm{Fe}^{2+}\right)$ in non-oxidizing oxygen environment. Upon oxidation magnetite $\left(\mathrm{Fe}_{3} \mathrm{O}_{4}\right)$ gets converted into maghemite $\left(\gamma \mathrm{Fe}_{2} \mathrm{O}_{3}\right)$.

$\mathrm{Fe}^{2+}+2 \mathrm{Fe}^{3+}+8 \mathrm{OH}-\mathrm{Fe}_{3} \mathrm{O}_{4}+4 \mathrm{H}_{2} \mathrm{O}$

Briefly, $0.2 \mathrm{M}$ Iron chloride hexa-hydrate $\left(\mathrm{FeCl}_{3} .6 \mathrm{H}_{2} \mathrm{O}\right)$ and 0.1 $M$ Iron sulphate hepta-hydrate $\left(\mathrm{FeSO}_{4} \cdot 7 \mathrm{H}_{2} \mathrm{O}\right)$ solutions were prepared in $150 \mathrm{ml}$ of deionised water. The resulting solution was stirred in a magnetic stirrer to obtain a homogenous mixture. Then $120 \mathrm{ml}$ of $1 \mathrm{M}$ sodium hydroxide $(\mathrm{NaOH})$ solution was added at the rate of $0.5 \mathrm{ml} / \mathrm{min}$. to the previous solution at $80^{\circ} \mathrm{C}$ at 800 rpm, until a black precipitate was formed. Change in the colour of the precursor solution from yellow to black is the preliminary confirmation of MNP synthesis. Addition of $\mathrm{NaOH}$ was stopped at this step and stirred for additional 30 mins. The black solution obtained was centrifuged at $10,000 \mathrm{rpm}$ for 10 minutes and washed with distilled water 5 times to get a black precipitate of
MNP. The pellet was dried at $60^{\circ} \mathrm{C}$ for 24 hours to get the powder of Iron oxide nanoparticles.

\section{Polymeric coating of magnetic nanoparticles}

The extracted carrageenan was used as a stabilizing agent for MNP to prevent oxidation and agglomeration of MNPs. 2:1 ratio of $\mathrm{FeCl}_{3} \cdot 6 \mathrm{H}_{2} \mathrm{O}$ and $\mathrm{FeSO}_{4} \cdot 7 \mathrm{H}_{2} \mathrm{O}$ were used for MNP synthesis as described above. To the precursor solution $20 \mathrm{ml}$ of carrageenan solution was added and the mixture for kept at $80^{\circ} \mathrm{C}$ at 800 rpm. $1 \mathrm{M}$ potassium hydroxide $(\mathrm{KOH})$ solution was added at the rate of $0.5 \mathrm{ml} / \mathrm{min}$ to the solution until a black precipitation is formed. The $\mathrm{pH}$ was 9.39 at the completion of reaction. The black precipitate was centrifuged at 10,000 rpm for 10 minutes and was washed with distilled water 5 times. The pellet was dried at $60^{\circ} \mathrm{C}$ for 24 hours to get the powder of surface modified carrageenan coated magnetic nano-particles (C-MNPs) [5,6].

\section{Characterization techniques}

Synthesized polymeric coated nanoparticles are characterized by various techniques [7-10]. The size of the synthesized C-MNPs was identified using scanning electron microscope (SEM). The elemental composition of synthesized C-MNPs was found through Energy Dispersive X-ray Spectroscopy (EDAX). Dynamic Light Scattering was used to find the hydrodynamic size and Zeta potential was done to find the stability of C-MNPs. The crystal structure of the synthesized MNPs was done using $X$ ray Diffraction technique.

\section{Haemolytic assay}

For drug delivery applications, the nanoparticles should be nontoxic and non-haemolytic. Hence the synthesized carrageenan coated nanoparticles were tested for their anti-haemolytic activity. Briefly, fresh goat blood was obtained from slaughter house, mixed with EDTA to avoid coagulation. $2 \mathrm{ml}$ of the blood was mixed with $4 \mathrm{ml}$ of phosphate buffer (PBS- $\mathrm{pH} 7.4$ ), centrifuged at $10000 \mathrm{rpm}$ for $5 \mathrm{~min}$ at $5^{\circ} \mathrm{C}$ to separate red blood cells (RBCs). The obtained pellet (RBCs) washed twice with $10 \mathrm{ml}$ of PBS and finally diluted to $20 \mathrm{ml}$ with PBS. $0.4 \mathrm{ml}$ of diluted RBC suspension was exposed to $1.6 \mathrm{ml}$ of the nanoparticle PBS suspension with the various concentrations to make the final nanoparticle concentration $2 \mathrm{ml}$. Distilled water and SDS were used as positive control and PBS was used as negative control. After incubation at room temperature for $1 \mathrm{~h}$, all the samples were centrifuged at $5000 \mathrm{rpm}$ for $10 \mathrm{~min}$ at $4^{\circ} \mathrm{C}$. Formation of clear supernatant and a red colored pellet indicates the anti-haemolytic activity [11].

\section{Results and Discussion}

The red algae rich in carrageenan were obtained from Rameswaram, Tamil Nadu, India. The samples were processed and dried into fine powder as shown in Figure 1. Alkaline extraction of carrageenan was done to get crude polymer solution. Alkali treatment results in breakdown of the sulphate bonds between the galactans unit of carrageenan. During extraction, $90 \%$ of the red algae were found to contain carrageenan after boiling the solution. The algal biomass was separated using ice cold ethanol precipitation and only $10 \%$ of biomass was obtained as wastage of the total content. The results indicate that Kappaphycus alvarezzi 


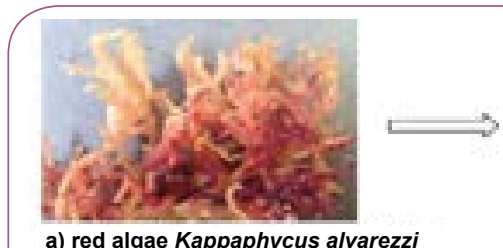

a) red algae Kappaphycus alvarezzi

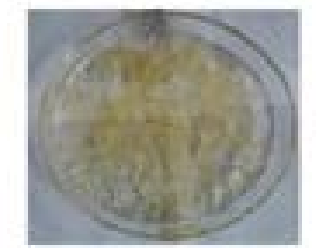

d) Dried carrageenan gel

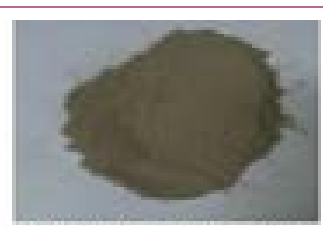

b) fine power of red algae 1

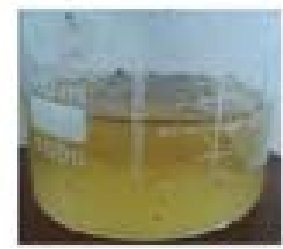

c) carrageenan in boiled water

Figure 1 Extraction of carrageenan from red algae.

is rich in natural polymer carrageenan and can be easily extracted from the biomass with simple steps. The obtained powdered carrageen polymer was used as a stabilizing agent for magnetic nanoparticles.

Preliminary confirmation on magnetic nanoparticle synthesis was done by visualizing the color change from reddish- yellow to reddish brown then to black colored solution. This color change is indicates the conversion of various oxidation phases of iron oxide molecules. The reddish brown color may be the phases of maghemite or hematite while the black color indicates the formation of magnetite nanoparticles. However, iron particles due to their higher oxidation may convert to maghemite upon continuous oxidation of magnetite nanoparticles. This will result in different forms of iron oxide nanoparticles. To prevent such oxidation different stabilizing or coating agents are used. Here, carrageen was used as a stabilizing agent. The polymer solution will form as a layer for each nanoparticle during synthesis resulting in inhibition of oxidation and agglomeration of synthesized magnetic nanoparticles. The resulting polymer coated magnetite nanoparticles (C-MNP) possess magnetic property and this was confirmed by placing a bar magnet near the sample. The magnetite particles moves immediately towards the bar magnet indicating their magnetic nature as shown in Figure 2.

The size of the synthesized nanoparticles was analyzed by SEM technique. The average size of the nanoparticles range from 70 $\mathrm{nm}$ to $100 \mathrm{~nm}$ as shown in the Figure 3. SEM image shows that the particles are spherical in shape. The size of the nanoparticles should be less than $100 \mathrm{~nm}$ and more than $10 \mathrm{~nm}$ for drug delivery applications. Hence, the synthesized C-MNPs are eligible for drug loading and delivering to the human body. Further, the hydrodynamic size of the nanoparticles was analyzed by Dynamic Light Scattering (DLS) method with Particle size analyzer. The average particle size distribution was found to be $140.5 \mathrm{~nm}$ (Figure 4). The size measured by DLS technique will usually be higher than that of the size obtained from SEM. The main reason is that DLS measures the hydrodynamic size of both the surrounding solvent molecules and the nanoparticles. Hence, the size is usually larger for DLS compared to the size obtained from SEM technique.

Zeta potential analysis was carried out to check the stability of C-MNPs. The stability was found to be $-26.3 \mathrm{mV}$ which shows moderate stability as shown in Figure 5. The result indicates that higher concentration of polymer is required to increase the stability of nanoparticles. The negative sign indicates that the surface of the nanoparticles was negatively charged which may be due to the charge of the carrageenan coated on magnetic nanoparticles. Figure 6 shows the diffraction peak analyzed from X-ray diffraction study. The synthesized C-MNPs are cubic centered with miller indices (311), (220), (222), (400), (422), (511) and (540) (Figure 6).

From the characterization studies it is clear that the polymer coated magnetic particles are suitable for drug delivery applications. However, nanoparticles when enters the human body cells it must be non-toxic. Hence to validate the toxicity, preliminary screening was done by haemolysis assay. Figure 7a represents the controls; distilled water, SDS and phosphate

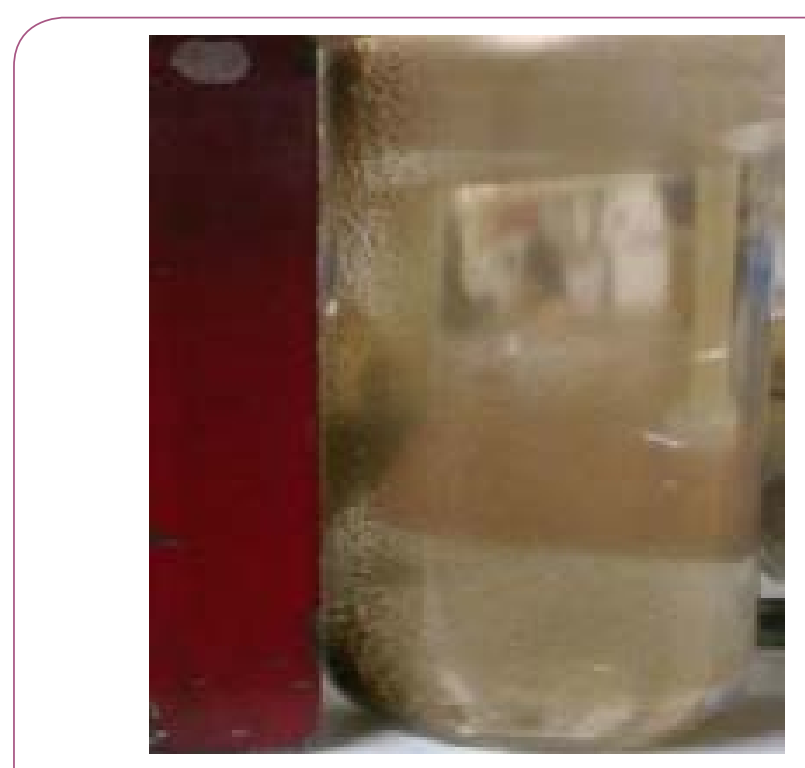

Figure 2 C-MNPs attracted towards bar magnet.

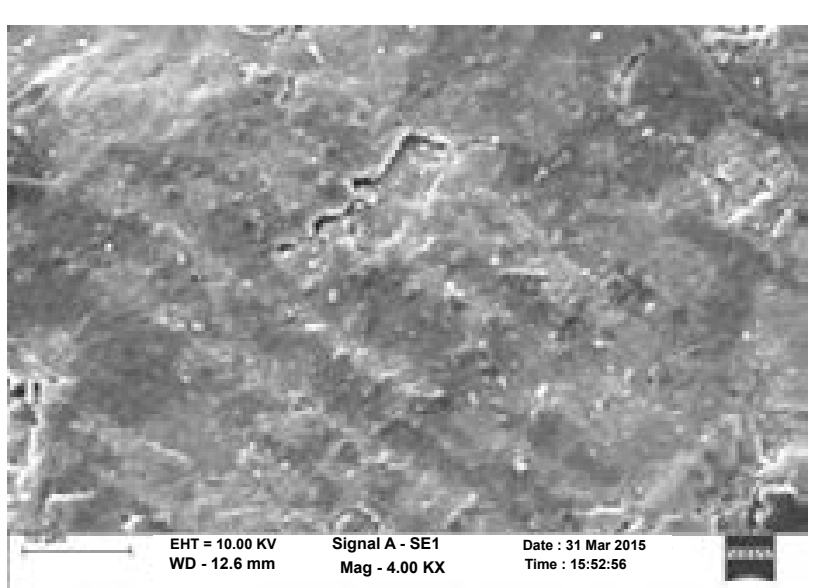

Figure 3 shows SEM image of C-MNP. 


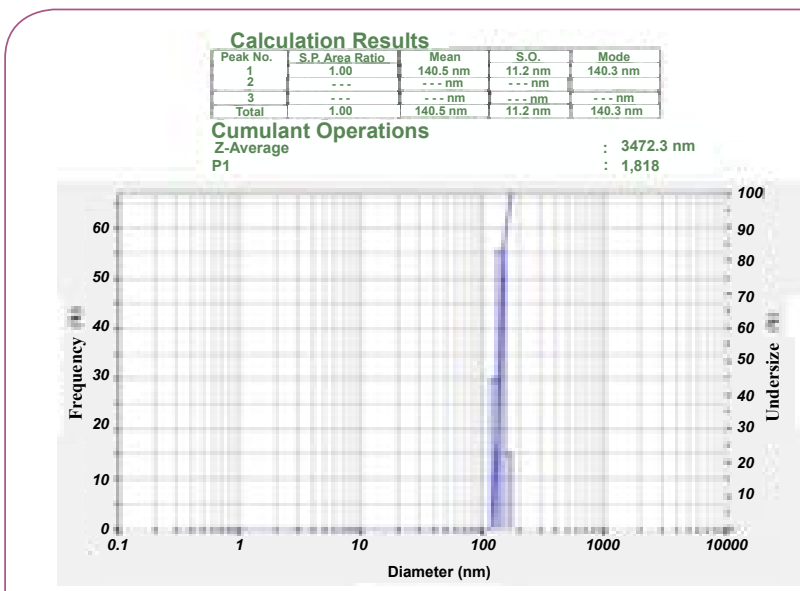

Figure 4 Hydrodynamic size observed through DLS technique.

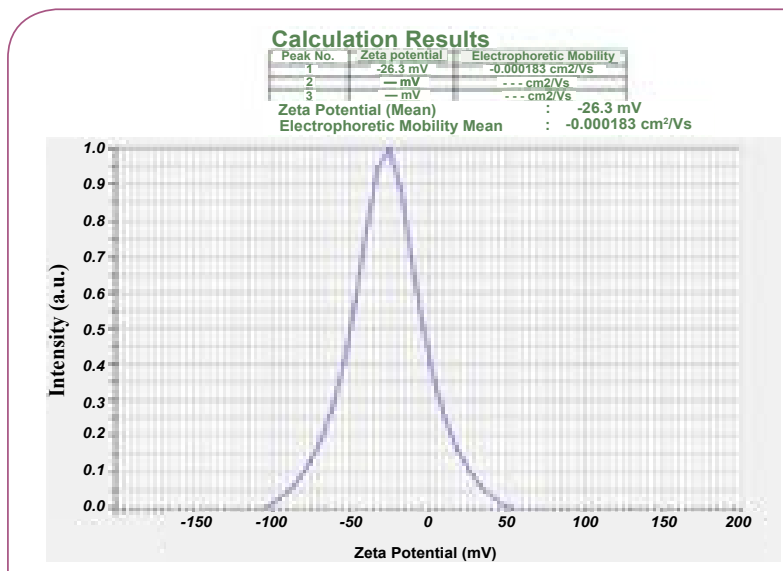

Figure 5 Zeta potential analysis to check the stability of C-MNPs.

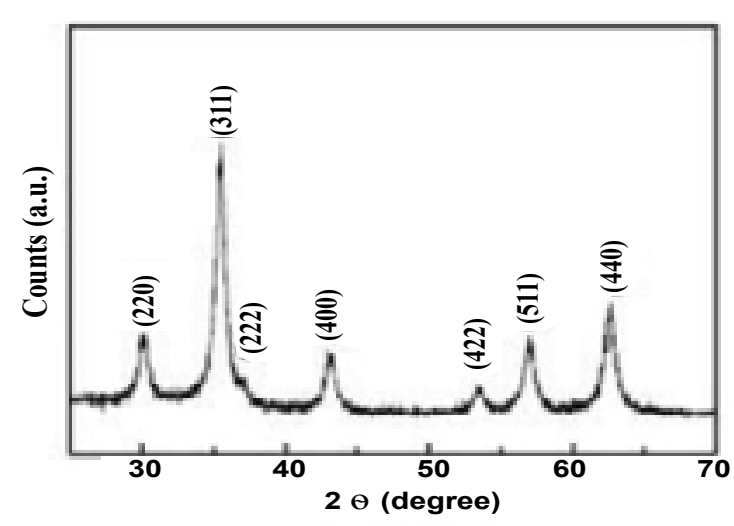

Figure 6 X-ray diffraction pattern of C-MNP.

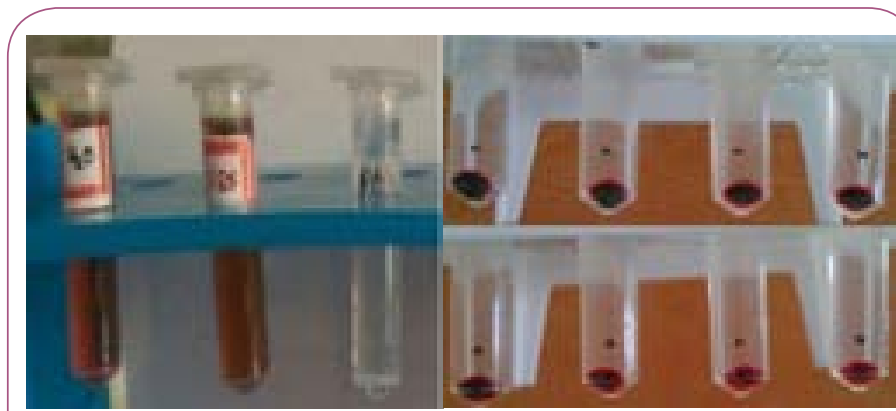

Figure 7 (a) represents the controls used for the assay and (b) represents the anti-haemolytic activity of synthesized C-MNPs. P1 to P8 indicates different concentrations of C-MNP (P1 - $4100 \mu \mathrm{g} / \mathrm{ml}, \mathrm{P} 2-2350 \mu \mathrm{g} / \mathrm{ml}, \mathrm{P} 3-1350 \mu \mathrm{g} /$ $\mathrm{ml}, \mathrm{P} 4-760 \mu \mathrm{g} / \mathrm{ml}, \mathrm{P} 5-440 \mu \mathrm{g} / \mathrm{ml}, \mathrm{P} 6-250 \mu \mathrm{g} / \mathrm{ml}, \mathrm{P} 7-$ $143 \mu \mathrm{g} / \mathrm{ml}, \mathrm{P} 8-81 \mu \mathrm{g} / \mathrm{ml}$.

buffer. From the figure it is clear that distilled water and SDS shows haemolytic activity. Distilled water undergoes osmolysis which results in rupture of red blood cells. SDS acts as a detergent and hence damages the cell wall of red blood cells resulting in haemolysis. Phosphate buffer shows anti-haemolytic activity and C-MNPs of different concentrations (Figure $\mathbf{7 b}$ ) shows antihaemolytic activity. The results indicate that C-MNPs are antihaemolytic even at higher concentration of $4100 \mu \mathrm{g} / \mathrm{ml}$ and hence can be used for drug delivery applications.

\section{Conclusion}

The present study reports on the extraction and coating of natural polymer carrageenan from red algae. Results obtained through various techniques confirm that the particles are in nanometer size. Haemolysis assay indicates that the synthesized C-MNPs can be used for drug delivery applications. Experiments on screening of C-MNPs such as anti-oxidant assay, drug adsorption and drug release studies will be carried out in the future for drug delivery applications. Application of natural polymers for surface coating of magnetic nanoparticles would be beneficial as they are nontoxic with higher bioavailability and degradability. 


\section{References}

1 Wei W, Quanguo H, Changzhong J (2008) Magnetic Iron Oxide Nanoparticles: Synthesis and Surface Functionalization Strategies, Nanoscale Res Lett 3: 397-415.

2 Tartaj P (2006) Synthesis, properties and biomedical applications of magnetic nanoparticles. Handbook of Magnetic Materials; Elsevier: Amsterdam, The Netherlands $403 \mathrm{p}$.

3 Estevez JM, Ciancia M, Cerezo AS (2000) The system of low-molecularweight carrageenans and agaroids from the room-temperatureextracted fraction of Kappaphycus alvarezii. Carbohydrate Research 325: 287-299.

4 Kandapal ND, Sah N, Losahli R, Joshni R, Prasad J (2013) Coprecipitation method of synthesis and characterization of iron oxide nanoparticles, J. of Sci Res73: 87-90.

5 Ana L, Daniel-da-Silva (2007) In Situ Synthesis of Magnetite Nanoparticles in Carrageenan Gels, Biomacromolecules 8: 23502357.
6 Raman M, Devi V, Doble M (2015) Biocompatible t-carrageenan- - maghemite nanocomposite for biomedical applications-synthesis, characterization and in vitro anticancer efficacy. Raman et al. Journal of Nanobiotechnology 13:18.

7 Doan Thi Kim (2009) Preparation and characterization of magnetic nanoparticles with chitosan coating, J. Phys Conf. Ser 187: 012036.

8 Lu AH, Salabas EL, Schüth F (2007) Magnetic nanoparticles: synthesis, protection, functionalization, and application. Angew Chem Int Ed 46: $1222-1244$.

9 Gupta AK, Gupta M (2005) Synthesis and surface engineering of iron oxide nanoparticles for biomedical applications. Biomaterials 25: 3995-4021.

10 Cumbat L, Greenleaf J, Leun D, SenGupta AK (2003) Polymer supported inorganic nanoparticles: characterization and environmental applications. React Funct Polym 54: 167-180.

11 Teodora M (2013) Hemolysis as Expression of Nanoparticles-Induced Cytotoxicity in Red Blood Cells. Biotechnology, Molecular Biology and Nanomedicine 1: 7-12. 